Pacific Journal of Mathematics

ON AN EXTENSION OF THE IKEHARA TAUBERIAN 


\title{
ON AN EXTENSION OF THE IKEHARA TAUBERIAN THEOREM
}

\section{JUNICHI ARAMAKI}

\begin{abstract}
A specific example of the Ikehara Tauberian theorem is extended to the case where the zeta function has a pole of order $p>1$ at the first singularity. And we have an application to asymptotic behavior of eigenvalues for some partial differential operator.
\end{abstract}

0. Introduction. In order to study the asymptotic behavior of eigenvalues for some differential or pseudodifferential operators, one frequently uses a specific example of Ikehara's Tauberian theorem. To be more precise, let $P$ be a positive definite self-adjoint operator on a separable Hilbert space $H$ with the domain of definition $K$ which is dense in $H$. If we denote the spectral resolution associated to $P$ by $\{E(\lambda)\}$, we can define complex powers of $P$ :

$$
P^{z}=\int_{0}^{\infty} \lambda^{z} d E(\lambda)
$$

where $\lambda^{z}$ for $\lambda>0$ take the principal values. If we assume that the canonical injection from $K$ which is equipped with the graph norm to $H$ is compact, it is well known that the spectrum $\sigma(P)$ of $P$ is discrete. This enables one to write the sequence of eigenvalues by $0<\lambda_{1} \leq \lambda_{2} \leq \cdots, \lambda_{k} \rightarrow \infty(k \rightarrow \infty)$ with repetition according to multiplicity and let $N(\lambda)$ be the counting function of eigenvalues: $N(\lambda)=\#\left\{j ; \lambda_{j} \leq \lambda\right\}$. If $\sum_{j=1}^{\infty} \lambda_{j}^{a}$ is convergent for some $a<0, P^{z}$ is of trace class and for $\operatorname{Re} z<a$,

$$
\operatorname{Tr} P^{z}=\sum_{j=1}^{\infty} \lambda_{j}^{z}
$$

Then a specific example of Ikehara's Tauberian theorem says:

Proposition 1. (Wiener [13] and Donoghue [5].) Let $\operatorname{Tr} P^{z}$ be holomorphic for $\operatorname{Re} z<a(<0)$. Assume that there exists a constant $A$ such that

$$
\operatorname{Tr} P^{z}-\frac{A}{z-a}
$$


is continuous for $\operatorname{Re} z \leq a$. Then we have

$$
N(\lambda)=\frac{A}{a} \lambda^{-a}(1+o(1)) \text { as } \lambda \rightarrow \infty .
$$

For realization $P$ in $H=L^{2}\left(\mathbf{R}^{n}\right)$ of elliptic differential or pseudodifferential operators, $\operatorname{Tr} P^{z}$ has a simple pole at the first singularity. Applying this proposition, we could obtain the asymptotic behavior of $N(\lambda)$. (See, for example, Seeley [11].) But there are some hypoelliptic operators where $\operatorname{Tr} P^{z}$ has a pole of order $p>1$ at the first singularity $s=a$. We refer the reader to, for example, Aramaki [1], [2], Mohamed [9] and Menikoff-Sjöstrand [8]. To get the first term for such operators we extended Proposition 1 as follows:

Proposition 2. ([1; Proposition 5.3].) Let $\operatorname{Tr} P^{z}$ be holomorphic for $\operatorname{Re} z<a(<0)$. Assume that there exist constants $A_{0}, A_{1}, \ldots, A_{p}$ such that

$$
\operatorname{Tr} P^{z}-\sum_{j=0}^{p} \frac{A_{j}}{(z-a)^{j}}
$$

is continuous for $\operatorname{Re} z \leq a$. Then we have

$$
N(\lambda)=\frac{(-1)^{p-1} A_{p}}{(p-1) ! a}(\log \lambda)^{p-1} \lambda^{-a}(1+o(1)) \quad \text { as } \lambda \rightarrow \infty .
$$

By this proposition, we could get the first term of $N(\lambda)$. However, we cannot find the coefficients of the term $(\log \lambda)^{j} \lambda^{-a}(j<p-1)$.

The purpose of this paper is to determine the coefficients $C_{j}$ of the asymptotic behavior of the form:

$$
N(\lambda)=\sum_{j=0}^{p-1} C_{j}(\log \lambda)^{j} \lambda^{-a}+O\left(\lambda^{-a-\delta}\right)
$$

for some $\delta>0$ as $\lambda \rightarrow \infty$. The proof is more complicated than that of Proposition 2 and essentially due to the inverse Mellin transformation. (cf. Duistermaat-Guillemin [6].)

The plan of this paper is as follows. In $\S 1$, we give the main theorem. Section 2 is devoted to the proof of the main theorem. Section 3 gives an example to illustrate our theory. Finally in Appendix, we shall discuss analytic continuation of a zeta function which is used in $\S 3$.

1. Statement. Let $H$ be a separable Hilbert space and $P$ a densely defined positive self-adjoint operator on $H$ with the domain of definition $K$. We regard $K$ equipped with the graph norm as a Hilbert 
space. We assume:

(H) The canonical injection from $K$ to $H$ is compact.

Since the domain of definition $K$ of $P$ is imbedded compactly to $H$, the spectrum $\sigma(P)$ of $P$ is discrete, i.e., both the following hold: $\lambda \in \sigma(P)$ is an isolated point of $\sigma(P)$. $\lambda \in \sigma(P)$ is an eigenvalue of finite multiplicity.

Thus we can denote the sequence of eigenvalues by $0<\lambda_{1} \leq \lambda_{2} \leq$ $\cdots, \lambda_{k} \rightarrow \infty(k \rightarrow \infty)$ with repetition according to multiplicity.

Since complex powers of $P$ is defined by $(0.1)$, we can define $\operatorname{Tr} P^{-s}$ which denotes the trace of $P^{-s}$ if $P^{-s}$ is of trace class.

Then we have:

THEOREM. Let $P$ be a positive self-adjoint operator on $H$ satisfying (H). Assume that

(i) $P^{-s}$ is of trace class for large $\operatorname{Re} s>0$ and $\operatorname{Tr} P^{-s}$ has a meromorphic extension $Z_{P}(s)$ in the complex plane $\mathbf{C}$ whose poles are distributed on the real line.

(ii) $Z_{P}(s)$ has the first singularity at $s=a(>0)$ and

$$
Z_{P}(s)-\sum_{j=1}^{p} \frac{A_{j}}{(j-1) !}\left(-\frac{d}{d s}\right)^{j-1} \frac{1}{s-a}
$$

is holomorphic in $\{s \in \mathbf{C} ; \operatorname{Re} s>a-\delta\}$ for some $\delta>0$.

(iii) $Z_{P}(s)$ is of polynomial order with respect to $\operatorname{Im} s$ in all vertical strips, excluding neighborhoods of the poles.

Then we have for some $\delta_{0}>0$,

$$
N_{P}(\lambda)=\left.\sum_{j=1}^{p} \frac{A_{j}}{(j-1) !}\left(\frac{d}{d s}\right)^{j-1}\left(\frac{\lambda^{s}}{s}\right)\right|_{s=a}+O\left(\lambda^{a-\delta_{0}}\right)
$$

as $\lambda \rightarrow+\infty$.

Here it is said that $s=a$ is the first singularity of $Z_{P}(s)$ if $Z_{P}(s)$ is holomorphic in $\{s \in \mathbf{C} ; \operatorname{Re} s>a-\delta\}$ for some $\delta>0$, except a pole at $s=a$.

2. Proof of Theorem. First of all, define $Q=P^{2 a}$, then the eigenvalues of $Q$ are $\mu_{j}=\lambda_{j}^{2 a}$. It easily follows that $Z_{Q}(s)=Z_{P}(2 a s)$ has 
the first singularity at $s=1 / 2$ and

$$
\begin{gathered}
Z_{Q}(s)-\sum_{j=1}^{p} \frac{B_{j}}{(j-1) !}\left(-\frac{d}{d s}\right)^{j-1} \frac{1}{s-1 / 2} \\
=Z_{Q}(s)-\sum_{j=1}^{p} \frac{B_{j}}{(s-1 / 2)^{j}}
\end{gathered}
$$

is holomorphic for $\operatorname{Re} s \geq 1 / 2-\delta / 2 a$ where $B_{j}=A_{j} /(2 a)^{j}$. Here we note that by Proposition $2, N_{Q}(\mu)=\#\left\{j ; \mu_{j} \leq \mu\right\}$ is of at most polynomial growth in $\mu$. This enables one to define, for $\operatorname{Re} z>0$,

$$
\Theta_{Q}(z)=\operatorname{Tr} e^{-z Q}=\sum_{j=1}^{\infty} e^{-z \mu_{j}}
$$

In fact, since

$$
j=N_{Q}\left(\mu_{j}\right) \sim \frac{B_{p}}{(p-1) !}\left(\log \mu_{j}\right)^{p-1} \mu_{j}^{1 / 2},
$$

there exists a constant $C$ such that $C j \leq \mu_{j}$ for large $j$. Thus it is clear that $(2.2)$ is well defined by noting the following inequality: for some $C^{\prime}>0$

$$
\sum_{j=1}^{\infty}\left|e^{-z \mu_{\jmath}}\right| \leq \sum_{j=1}^{\infty} e^{-C j \operatorname{Re} z} \leq C^{\prime}(\operatorname{Re} z)^{-2} \sum_{j=1}^{\infty} j^{-2}<\infty .
$$

By the inverse Mellin transformation, $\Theta_{Q}(z)$ and $Z_{Q}(s)$ can be related to each other: For $\operatorname{Re} z>0$,

$$
\Theta_{Q}(z)=\frac{1}{2 \pi i} \int_{\operatorname{Re} s=c} z^{-s} Z_{Q}(s) \Gamma(s) d s
$$

where $\Gamma(s)$ is the $\Gamma$-function:

$$
\Gamma(s)=\int_{0}^{\infty} e^{-t} t^{s-1} d t
$$

and $c>0$ is sufficiently large (cf. [6]).

Since $\Gamma(s)$ is exponentially decreasing as $\operatorname{Im} s \rightarrow+\infty$ in all vertical strips, excluding neighborhoods of the poles, it follows from (iii) that $Z_{Q}(s) \Gamma(s)$ is also exponentially decreasing in all vertical strips, excluding neighborhoods of the poles of $Z_{Q}(s)$ and $\Gamma(s)$. This allows one to shift the path of integration in (2.3) by $c \searrow c_{0}$ where $1 / 2-\delta / 4 a<c_{0}<1 / 2$. Thus we can rewrite $\Theta_{Q}(z)$ into the form:

$$
\Theta_{Q}(z)=\sum_{j=1}^{p} B_{j} I_{j}(z)+R_{c_{0}}(z)
$$


where

$$
\begin{gathered}
I_{j}(z)=\frac{1}{2 \pi i} \int_{|s-1 / 2|=\varepsilon} \frac{z^{-s} \Gamma(s)}{(s-1 / 2)^{j}} d s \text { and } \\
R_{c_{0}}(z)=\frac{1}{2 \pi i} \int_{\operatorname{Re} s=c_{0}} z^{-s} Z_{Q}(s) \Gamma(s) d s .
\end{gathered}
$$

Here $\varepsilon$ satisfies $0<\varepsilon<\delta / 2 a$. We see from the Cauchy theorem that

$$
I_{j}(z)=\left.\frac{1}{(j-1) !}\left(\frac{d}{d s}\right)^{j-1}\left\{z^{-s} \Gamma(s)\right\}\right|_{s=1 / 2} .
$$

Consequently $\Theta_{Q}(z)$ is reformed in the form

$$
\Theta_{Q}(z)=\left.\sum_{j=1}^{p} \frac{B_{j}}{(j-1) !}\left(\frac{d}{d s}\right)^{j-1}\left\{z^{-s} \Gamma(s)\right\}\right|_{s=1 / 2}+R_{\mathcal{C}_{0}}(z) .
$$

Now we choose $\rho \in S(\mathbf{R})$ so that $F \rho$ is an even function with compact support and $(F \rho)(0)=1, \rho(0)>0, \rho \geq 0$ where $S(\mathbf{R})$ is the Schwartz space of smooth rapidly decreasing functions on $\mathbf{R}$ and $F \rho$ means the Fourier transformation of $\rho$ :

$$
(F \rho)(t)=\int_{-\infty}^{\infty} e^{-i t \tau} \rho(\tau) d \tau
$$

By the Lebesgue theorem and the definition of $N_{Q}(\tau)$, we have

$$
\begin{aligned}
I(\mu) & =\int_{-\infty}^{\infty} \rho(\mu-\tau) d N_{Q}(\tau)=\lim _{\varepsilon \downarrow 0} \int_{-\infty}^{\infty} e^{-\varepsilon \tau} \rho(\mu-\tau) d N_{Q}(\tau) \\
& =\lim _{\varepsilon \downarrow 0} \sum_{j=1}^{\infty} e^{-\varepsilon \mu_{J}} \rho\left(\mu-\mu_{j}\right) \\
& =\lim _{\varepsilon \downarrow 0}(2 \pi)^{-1} \sum_{j=1}^{\infty} \int_{-\infty}^{\infty} e^{-(\varepsilon+i t) \mu_{J}}(F \rho)(t) e^{i \mu t} d t \\
& =\lim _{\varepsilon \downarrow 0}(2 \pi)^{-1} \int_{-\infty}^{\infty} \Theta_{Q}(\varepsilon+i t)(F \rho)(t) e^{i \mu t} d t \\
& =\sum_{j=1}^{p} \frac{B_{j}}{(j-1) !} I_{j}^{0}(\mu)+R_{\mathcal{c}_{0}}^{0}(\mu)
\end{aligned}
$$

where

$$
I_{j}^{0}(\mu)=\left.\lim _{\varepsilon \downarrow 0}(2 \pi)^{-1} \int_{-\infty}^{\infty}\left(\frac{d}{d s}\right)^{j-1}\left\{(\varepsilon+i t)^{-s} \Gamma(s)\right\}\right|_{s=1 / 2}(F \rho)(t) e^{i \mu t} d t
$$


and

$$
R_{c_{0}}^{0}(\mu)=\lim _{\varepsilon \downarrow 0}(2 \pi)^{-1} \int_{-\infty}^{\infty} R_{c_{0}}(\varepsilon+i t)(F \rho)(t) e^{i \mu t} d t .
$$

In the sequel, we shall study the asymptotic behavior of $I_{j}^{0}(\mu)$ and $R_{c_{0}}^{0}(\mu)$ as $\mu \rightarrow+\infty$. In order to do so, we prove the following four lemmas.

LEMMA 2.1. Let $s \in B_{r}(1 / 2)=\{s \in \mathbf{C} ;|s-1 / 2| \leq r\}$. Then for every integer $j \geq 0$ and $0<r<1 / 2$,

$$
\lim _{\varepsilon \downarrow 0} \int_{-\infty}^{\infty}\left(\frac{d}{d s}\right)^{j}(\varepsilon+i t)^{-s} e^{i \mu t} d t=\int_{-\infty}^{\infty}\left(\frac{d}{d s}\right)^{j}(i t)^{-s} e^{i \mu t} d t
$$

Moreover the integral in the right-hand side is uniformly convergent on $B_{r}(1 / 2)$.

Proof. Since $(d / d s)^{j}(\varepsilon+i t)^{-s}=(\varepsilon+i t)^{-s}(-\log (\varepsilon+i t))^{j}$, it suffices to prove that:

$$
\text { (2.8) } \lim _{\varepsilon \downarrow 0} \int_{-\infty}^{\infty}(\varepsilon+i t)^{-s}(\log (\varepsilon+i t))^{j} e^{i \mu t} d t=\int_{-\infty}^{\infty}(i t)^{-s}(\log (i t))^{j} e^{i \mu t} d t
$$

and the integral in the right-hand side in (2.8) is uniformly convergent on $B_{r}(1 / 2)$. By virtue of the mean value theorem, there exists $\theta \in$ $(0,1)$ such that

$$
\begin{aligned}
(\varepsilon+ & i t)^{-s}(\log (\varepsilon+i t))^{j} \\
& =(i t)^{-s}(\log (i t))^{j} \\
& +\varepsilon \int_{0}^{1}(\varepsilon \theta+i t)^{-s-1}\left\{-s(\log (\varepsilon \theta+i t))^{j}+j(\log (\varepsilon \theta+i t))^{j-1}\right\} d \theta .
\end{aligned}
$$

If we choose $\delta>0$ so that $r+\delta<1 / 2$, there exists a constant $C$ independent of $\varepsilon$ and $s \in B_{r}(1 / 2)$ such that

$$
\begin{aligned}
\left|(\varepsilon \theta+i t)^{-s-1}(\log (\varepsilon \theta+i t))^{k}\right| \leq C|t|^{-\operatorname{Re} s-1+\delta} \leq C|t|^{-3 / 2+r+\delta}, & \\
& (k=j \text { or } k=j-1)
\end{aligned}
$$

for all $|t| \geq 1$. So we have

$$
\begin{gathered}
\varepsilon \int_{|t| \geq 1}\left|\int_{0}^{1}(\varepsilon \theta+i t)^{-s-1}(\log (\varepsilon \theta+i t))^{k} d \theta\right| d t \\
\leq \varepsilon C \int_{|t| \geq 1}|t|^{-3 / 2+r+\delta} d t \rightarrow 0
\end{gathered}
$$


as $\varepsilon \downarrow 0$. On the other hand, if we choose $\delta$ so that $0<2 \delta<1 / 2-r$, then

$$
\begin{aligned}
& \varepsilon \int_{|t| \leq 1}\left|\int_{0}^{1}(\varepsilon \theta+i t)^{-s-1}(\log (\varepsilon \theta+i t))^{k} d \theta\right| d t \\
& \quad \leq \varepsilon \int_{|t| \leq 1} \int_{0}^{1}(\varepsilon \theta+|t|)^{\delta-1}(\varepsilon \theta+|t|)^{-r-2 \delta-1 / 2} d \theta d t \\
& \quad \leq \varepsilon \int_{0}^{1}(\varepsilon \theta)^{\delta-1} d \theta \int_{|t| \leq 1}|t|^{-r-2 \delta-1 / 2} d t \rightarrow 0
\end{aligned}
$$

as $\varepsilon \downarrow 0$. This completes the proof.

REMARK 2.2. By the above lemma, we have $0<b<1$ and every $k=0,1, \ldots$,

$$
\left.\int_{-\infty}^{\infty}\left(\frac{d}{d s}\right)^{k}(i t)^{-s}\right|_{s=b} e^{i \mu t} d t=\left.\left(\frac{d}{d s}\right)^{k} \int_{-\infty}^{\infty}(i t)^{-s} e^{i \mu t} d t\right|_{s=b}
$$

LEMMA 2.3. Let $0<b<1$. Then we have the following:

(2.10) $\left.\quad \lim _{\varepsilon \downarrow 0} \int_{-\infty}^{\infty}\left(\frac{d}{d s}\right)^{j-1}\left\{(\varepsilon+i t)^{-s} \Gamma(s)\right\}\right|_{s=b}(F \rho)(t) e^{i \mu t} d t$

$$
\begin{aligned}
= & \left.\int_{-\infty}^{\infty}\left(\frac{d}{d s}\right)^{j-1}\left\{(i t)^{-s} \Gamma(s)\right\}\right|_{s=b} e^{i \mu t} d t \\
& +O\left(\mu^{-1}\right) \text { as } \mu \rightarrow+\infty .
\end{aligned}
$$

Proof. Since

$$
\left.\left(\frac{d}{d s}\right)^{j-1}\left\{(\varepsilon+i t)^{-s} \Gamma(s)\right\}\right|_{s=b}
$$

is a linear combination of

$$
(\varepsilon+i t)^{-b}(\log (\varepsilon+i t))^{k}, \quad(0 \leq k \leq j-1),
$$

it suffices to prove:

(2.11) $\lim _{\varepsilon \downarrow 0} \int_{-\infty}^{\infty}(\varepsilon+i t)^{-b}(\log (\varepsilon+i t))^{k}(F \rho)(t) e^{i \mu t} d t$

$$
=\int_{-\infty}^{\infty}(i t)^{-b}(\log (i t))^{k} e^{i \mu t} d t+O\left(\mu^{-1}\right) \quad \text { as } \mu \rightarrow+\infty
$$


The integration by parts leads to

$$
\begin{aligned}
I_{k}^{0}(\mu ; \varepsilon)=\int_{-\infty}^{\infty}(\varepsilon+i t)^{-b}(\log (\varepsilon+i t))^{k}(F \rho)(t) e^{i \mu t} d t \\
=-\frac{1}{i \mu} \int_{-\infty}^{\infty} \frac{d}{d t}\left\{(\varepsilon+i t)^{-b}(\log (\varepsilon+i t))^{k}(F \rho)(t)\right\} e^{i \mu t} d t \\
=\frac{b}{\mu} \int_{-\infty}^{\infty}(\varepsilon+i t)^{-b-1}(\log (\varepsilon+i t))^{k}(F \rho)(t) e^{i \mu t} d t \\
\quad-\frac{k}{\mu} \int_{-\infty}^{\infty}(\varepsilon+i t)^{-b-1}(\log (\varepsilon+i t))^{k-1}(F \rho)(t) e^{i \mu t} d t \\
\quad-\frac{1}{i \mu} \int_{-\infty}^{\infty}(\varepsilon+i t)^{-b}(\log (\varepsilon+i t))^{k}(F \rho)^{\prime}(t) e^{i \mu t} d t
\end{aligned}
$$

Here $(F \rho)^{\prime}$ denotes the derivative of $F \rho$. We first estimate the third term of (2.12). Noting that for arbitrary $\delta(0<\delta<1-b)$ there exists a constant $C>0$ independent of $\varepsilon$ such that

$$
\mid(\varepsilon+i t)^{-b}\left(\left.\log (\varepsilon+i t)^{k}(F \rho)^{\prime}(t) e^{i \mu t}|\leq C| t\right|^{-b-\delta} \text { in supp }(F \rho)^{\prime},\right.
$$

it is easily seen that the third term is of $O\left(\mu^{-1}\right)$ as $\mu \rightarrow+\infty$ uniformly when $\varepsilon \downarrow 0$.

Next, we consider the first and second terms of (2.12). Since we may suppose $\operatorname{supp}(F \rho) \subset(-N, N)$ for some $N>0$, we can write

$$
\begin{aligned}
\int_{-\infty}^{\infty} & (\varepsilon+i t)^{-b-1}(\log (\varepsilon+i t))^{k}(F \rho)(t) e^{i \mu t} d t \\
= & \int_{-\infty}^{\infty}(\varepsilon+i t)^{-b-1}(\log (\varepsilon+i t))^{k} e^{i \mu t} d t \\
& +\int_{-N}^{N}(\varepsilon+i t)^{-b-1}(\log (\varepsilon+i t))^{k}((F \rho)(t)-1) e^{i \mu t} d t \\
& -\int_{|t| \geq N}(\varepsilon+i t)^{-b-1}(\log (\varepsilon+i t))^{k} e^{i \mu t} d t
\end{aligned}
$$

Since $(F \rho)(0)=1,|(F \rho)(t)-1| \leq M|t|$ for some $M>0$. Thus, taking $\delta>0$ small enough, there exist constants $C$ and $C^{\prime}$ independent of $\varepsilon$ such that

$$
\begin{gathered}
\int_{-N}^{N}\left|(\varepsilon+i t)^{-b-1}(\log (\varepsilon+i t))^{k}((F \rho)(t)-1) e^{i \mu t}\right| d t \\
\leq C \int_{-N}^{N}|t|^{-b-\delta} d t \leq C^{\prime} .
\end{gathered}
$$

Similarly taking $\delta>0$ small enough shows that we also have $\int_{|t| \geq N}\left|(\varepsilon+i t)^{-b-1}(\log (\varepsilon+i t))^{k} e^{i \mu t}\right| d t \leq C \int_{|t| \geq N}|t|^{-b-1+\delta} d t \leq C^{\prime}$. 
Hence we see that the second and third terms in the right-hand side in (2.13) are of $O(1)$ as $\mu \rightarrow+\infty$ uniformly in $\varepsilon$. Now, the integration by parts yields that

$$
\begin{aligned}
K_{k}(\mu ; \varepsilon) & =\int_{-\infty}^{\infty}(\varepsilon+i t)^{-b-1}(\log (\varepsilon+i t))^{k} e^{i \mu t} d t \\
& =\frac{1}{b i} \int_{-\infty}^{\infty}(\varepsilon+i t)^{-b} \frac{d}{d t}\left\{(\log (\varepsilon+i t))^{k} e^{i \mu t}\right\} d t \\
& =\frac{k}{b} K_{k-1}(\mu ; \varepsilon)+\frac{\mu}{b} M_{k}(\mu ; \varepsilon)
\end{aligned}
$$

where

$$
M_{k}(\mu ; \varepsilon)=\int_{-\infty}^{\infty}(\varepsilon+i t)^{-b}(\log (\varepsilon+i t))^{k} e^{i \mu t} d t
$$

Since $K_{0}(\mu ; \varepsilon)=(\mu / b) M_{0}(\mu ; \varepsilon)$, we have, by induction,

$$
K_{k}(\mu ; \varepsilon)=\frac{\mu}{b}\left[\sum_{s=0}^{k} b^{s-k} \frac{k !}{s !} M_{s}(\mu ; \varepsilon)\right] .
$$

Therefore, taking (2.12) and (2.13) into consideration, we have

$$
\begin{gathered}
I_{k}^{0}(\mu ; \varepsilon) \equiv \frac{b}{\mu} K_{k}(\mu ; \varepsilon)-\frac{k}{\mu} K_{k-1}(\mu ; \varepsilon)=M_{k}(\mu ; \varepsilon) \\
=\int_{-\infty}^{+\infty}(\varepsilon+i t)^{-b}(\log (\varepsilon+i t))^{k} e^{i \mu t} d t
\end{gathered}
$$

modulo $O\left(\mu^{-1}\right)$ uniformly when $\varepsilon \downarrow 0$. Finally it only remains to apply Lemma 2.1 (cf. (2.8)). This completes the proof.

LEMMA 2.4. Let $s$ be a complex number so that $0<\operatorname{Re} s<1$ and $\mu$ a positive real number. Then we have

$$
\int_{-\infty}^{\infty}(i t)^{-s} e^{i \mu t} d t=2 \sin s \pi \Gamma(1-s) \mu^{s-1}
$$

Proof. We first consider the integral

$$
I^{+}(s)=\int_{0}^{\infty}(i t)^{-s} e^{i \mu t} d t .
$$

The change of variable $\mu t \rightarrow t$ leads to

$$
I^{+}(s)=i^{-s} \mu^{s-1} \int_{0}^{\infty} t^{-s} e^{i t} d t .
$$

If we put $z=r e^{i \theta}, 0<\theta \leq \pi / 2$, we have

$$
\left|z^{-s} e^{i z}\right| \leq r^{-\operatorname{Re} s} e^{\theta \operatorname{Im} s} e^{-r \sin \theta}
$$


Since $\sin \theta>0$ in $(0, \pi / 2]$ and $z^{-s} e^{i z}$ is holomorphic function of $z=r e^{i \theta}$ in $0<\theta \leq \pi / 2$, we can deform the integral as follows:

$$
\begin{aligned}
I^{+}(s) & =i^{-s} \mu^{s-1} \int_{0}^{\infty}(i t)^{-s} e^{-t} i d t \\
& =i^{-2 s+1} \mu^{s-1} \int_{0}^{\infty} t^{-s} e^{-t} d t=i^{-2 s+1} \mu^{s-1} \Gamma(1-s) .
\end{aligned}
$$

If we put $z=r e^{i \theta},-\pi / 2 \leq \theta<0$, it follows from the same argument that

$$
I^{-}(s)=\int_{-\infty}^{0}(i t)^{-s} e^{i \mu t} d t=(-i)^{-2 s+1} \mu^{s-1} \Gamma(1-s) .
$$

Therefore

$$
\begin{aligned}
I^{+}(s)+I-(s) & =i\left\{i^{-2 s}-(-i)^{-2 s}\right\} \mu^{s-1} \Gamma(1-s) \\
& =2 \sin s \pi \Gamma(1-s) \mu^{s-1}
\end{aligned}
$$

This completes the proof.

Finally we consider the asymptotic behavior of the remainder term $R_{c_{0}}^{0}(\mu)$.

LEMMA 2.5. There exists $\delta>0$ such that $R_{c_{0}}^{0}(\mu)=O\left(\mu^{-1 / 2-\delta}\right)$ as $\mu \rightarrow \infty$.

Proof. If $Z_{Q}(s) \Gamma(s)$ has a pole at $s=s_{0}$ such that $0<s_{0}<c_{0}<1 / 2$, the above lemmas show that there exist some $\delta>0$ and $c_{1}\left(0<c_{1}<s_{0}\right)$ such that $R_{c_{0}}^{0}(\mu)=R_{c_{1}}^{0}(\mu)+O\left(\mu^{-1 / 2-\delta}\right)$. Thus in the definition (2.4) of $R_{c_{0}}(z)$ we may assume that $c_{0}>0$ is arbitrary. Moreover, if $Z_{Q}(s) \Gamma(s)$ has a pole at $s=0$, there exist some $d<0$ and sufficiently small $\varepsilon>0$ such that $R_{c_{0}}(z)=R^{\prime}(z)+R_{d}(z)$ where

$$
R^{\prime}(z)=\frac{1}{2 \pi i} \int_{|s|=\varepsilon} z^{-s} Z_{Q}(s) \Gamma(s) d s .
$$

We show that there exists $\delta>0$ such that

$$
R_{0}^{\prime}(\mu)=\lim _{\varepsilon \downarrow 0} \int_{-\infty}^{\infty} R^{\prime}(\varepsilon+i t)(F \rho)(t) e^{i \mu t} d t=O\left(\mu^{-1 / 2-\delta}\right)
$$

as $\mu \rightarrow \infty$. In fact, by the preceding arguments, it suffices to prove that

$$
\int_{-N}^{N}(\log t)^{j}(F \rho)(t) e^{i \mu t} d t=O\left(\mu^{-1 / 2-\delta}\right)
$$


as $\mu \rightarrow \infty$. For brevity we only consider the integral

$$
\begin{aligned}
& J_{j}(\mu)=\int_{0}^{N}(\log t)^{j}(F \rho)(t) e^{i \mu t} d t=J_{j}^{1}(\mu)+J_{j}^{2}(\mu) \text { where } \\
& J_{j}^{1}(\mu)=\int_{0}^{1 / \mu}(\log t)^{j}(F \rho)(t) e^{i \mu t} d t \quad \text { and } \\
& J_{j}^{2}(\mu)=\int_{1 / \mu}^{N}(\log t)^{j}(F \rho)(t) e^{i \mu t} d t .
\end{aligned}
$$

Since $(F \rho)(t)=(F \rho)(0)+t(F \rho)^{\prime}(\theta t), 0<\theta<1$, we have

$$
J_{j}^{1}(\mu)=\int_{0}^{1 / \mu}(\log t)^{j}\left(1+t(F \rho)^{\prime}(\theta t)\right) e^{i \mu t} d t .
$$

For any $a \in(0,1)$, there exist constants $C$ and $C^{\prime}>0$ such that

$$
\left|\int_{0}^{1 / \mu}(\log t)^{j} e^{i \mu t} d t\right| \leq C \int_{0}^{1 / \mu} t^{-a} d t \leq C^{\prime} \mu^{a-1}
$$

And

$$
\left|\int_{0}^{1 / \mu}(\log t)^{j} t(F \rho)^{\prime}(\theta t) e^{i \mu t} d t\right| \leq C \int_{0}^{1 / \mu} d t \leq C^{\prime} \mu^{-1} .
$$

Thus we see that $J_{j}^{1}(\mu)=O\left(\mu^{-\delta-1 / 2}\right)$. Next, by the integration by parts, we have

$$
\begin{aligned}
J_{j}^{2}(\mu)=\frac{i}{\mu}\left[\int_{1 / \mu}^{N} j t^{-1}(\log t)^{j-1}(F \rho)(t) e^{i \mu t} d t\right. \\
\left.\quad+\int_{1 / \mu}^{N}(\log t)^{j}(F \rho)^{\prime}(t) e^{i \mu t} d t\right] .
\end{aligned}
$$

For any $a>0$, we have with a constant $C>0$,

$$
\begin{aligned}
& \left|\int_{1 / \mu}^{N} j t^{-1}(\log t)^{j-1}(F \rho)(t) e^{i \mu t} d t\right| \\
& \quad \leq C \int_{1 / \mu}^{N} t^{-1-a} d t=O\left(\mu^{a}\right) \text { as } \mu \rightarrow \infty .
\end{aligned}
$$

It is clear that the second term in the parenthesis of (2.16) is of $O(1)$. Thus for some $\delta>0, J_{j}^{2}(\mu)=O\left(\mu^{-\delta-1 / 2}\right)$ as $\mu \rightarrow \infty$. Consequently it follows that for some $\delta>0, R_{0}(\mu)=O\left(\mu^{-\delta-1 / 2}\right)$ as $\mu \rightarrow \infty$. Thus we are reduced to prove that for some $d<0, R_{d}^{0}(\mu)=O\left(\mu^{-1 / 2-\delta}\right)$ as $\mu \rightarrow \infty$. But this fact follows from the same arguments in [3] (cf. [6]). This completes the proof. 
End of the proof of Theorem.

By virtue of the above lemmas, we have, modulo $O\left(\mu^{-1 / 2-\delta}\right)$ for some $\delta>0$ as $\mu \rightarrow+\infty$,

$$
\begin{aligned}
I(\mu) & =\int_{-\infty}^{\infty} \rho(\mu-\tau) d N_{Q}(\tau) \\
& \left.\equiv \sum_{j=1}^{p} \frac{B_{j}}{(j-1) !}(2 \pi)^{-1} \int_{-\infty}^{\infty}\left(\frac{d}{d s}\right)^{j-1}\left\{(i t)^{-s} \Gamma(s)\right\}\right|_{s=1 / 2} e^{i \mu t} d t .
\end{aligned}
$$

Here, taking Remark 2.2 and Lemma 2.4 into consideration,

$$
\begin{aligned}
I(\mu) & \left.\equiv \sum_{j=1}^{p} \frac{B_{j}}{(j-1) !}(2 \pi)^{-1}\left(\frac{d}{d s}\right)^{j-1}\left\{\Gamma(s) \int_{-\infty}^{\infty}(i t)^{-s} e^{i \mu t} d t\right\}\right|_{s=1 / 2} \\
& \left.\equiv \sum_{j=1}^{p} \frac{B_{j}}{(j-1) !}(2 \pi)^{-1}\left(\frac{d}{d s}\right)^{j-1}\left\{2 \sin s \pi \Gamma(s) \Gamma(1-s) \mu^{s-1}\right\}\right|_{s=1 / 2} .
\end{aligned}
$$

By the well known equation: $\sin s \pi \Gamma(s) \Gamma(1-s)=\pi$, we have

$$
I(\mu)=\left.\sum_{j=1}^{p} \frac{B_{j}}{(j-1) !}\left(\frac{d}{d s}\right)^{j-1}\left(\mu^{s-1}\right)\right|_{s=1 / 2}+O\left(\mu^{-1 / 2-\delta}\right) .
$$

Now it follows from Helffer [7] that there exists a constant $C$ such that

$$
\int_{-\infty}^{1} \int_{-\infty}^{+\infty} \rho(\mu-\tau) d N_{Q}(\tau) d \mu \leq C
$$

Thus we have

$$
\begin{aligned}
N_{Q}(\lambda) & =\int_{-\infty}^{\lambda} I(\mu) d \mu+O\left(\lambda^{1 / 2-\delta}\right) \\
& =\left.\sum_{j=1}^{p} \frac{B_{j}}{(j-1) !}\left(\frac{d}{d s}\right)^{j-1}\left(\frac{\mu^{s}}{s}\right)\right|_{s=1 / 2}+O\left(\lambda^{1 / 2-\delta}\right) .
\end{aligned}
$$

Noting that $N_{P}(\lambda)=N_{Q}\left(\lambda^{2 a}\right)$ and $B_{s}=A_{s} /(2 a)^{s}$ we have for some $\delta_{0}>0$,

$$
\begin{aligned}
N_{P}(\lambda) & =\left.\sum_{j=1}^{p} \frac{A_{j}}{(j-1) !}(2 a)^{-j}\left(\frac{d}{d s}\right)^{j-1}\left(\frac{\lambda^{2 a s}}{s}\right)\right|_{s=1 / 2}+O\left(\lambda^{a-\delta_{0}}\right) \\
& =\left.\sum_{j=1}^{p} \frac{A_{j}}{(j-1) !}\left(\frac{d}{d s}\right)^{j-1}\left(\frac{\lambda^{s}}{s}\right)\right|_{s=a}+O\left(\lambda^{a-\delta_{0}}\right) .
\end{aligned}
$$

This completes the proof of Theorem. 
3. Example. In this section we shall give an example. Let

$$
A=-\frac{\partial^{2}}{\partial x^{2}}-\frac{\partial^{2}}{\partial y^{2}}+\left(1+x^{2}\right) y^{2} \text { on } \mathbf{R}^{2} .
$$

By the celebrated Kato theorem, it follows that $A$ is an essentially self-adjoint operator on $L^{2}\left(\mathbf{R}^{2}\right)$, i.e., $A$ has a unique self-adjoint extension $P$ of $A$ as unbounded operator on $L^{2}\left(\mathbf{R}^{2}\right)$. Moreover $P$ is semi-bounded from below. By Robert [10] (cf. [4]), we can regard $P$ as a $L^{2}(\mathbf{R})$-valued operator as follows. If we define

$$
K=\left\{u \in L^{2}(\mathbf{R}) ;\left(-\frac{d^{2}}{d y^{2}}+y^{2}\right) u \in L^{2}(\mathbf{R})\right\} \quad \text { and } \quad H=L^{2}(\mathbf{R}),
$$

we see that

$$
Q(x)=-\frac{d^{2}}{d y^{2}}+\left(1+x^{2}\right) y^{2} \in L(K, H)
$$

where $L(K, H)$ denotes the Banach space of all bounded linear operators from $K$ to $H$. Thus we can regard $A$ as a $L^{2}(\mathbf{R})$-valued operator with the Weyl symbol

$$
\sigma_{W}(A)=\xi^{2}+Q(x) \in L(K, H) .
$$

Since $-d^{2} / d y^{2}+y^{2}$ has the complete set of the eigenvalues $\mu_{j}=2 j-1$ $(j=1,2, \ldots)$ of multiplicity one, ones of $\sigma_{W}(A)$ are given by

$$
\xi^{2}+\left(1+x^{2}\right)^{1 / 2} \mu_{j}
$$

It follows from [4] that

$$
\operatorname{Tr} P^{-s}-(2 \pi)^{-1} \iint \operatorname{Tr}\left(\xi^{2}+Q(x)\right)^{-s} d x d \xi
$$

is holomorphic for $\operatorname{Re} s>3 / 2-\delta$ for some $\delta>0$. Thus we are reduced to study

$$
\begin{aligned}
I(s) & =(2 \pi)^{-1} \iint \operatorname{Tr}\left(\xi^{2}+Q(x)\right)^{-s} d x d \xi \\
& =\sum_{j=1}^{\infty}(2 \pi)^{-1} \iint\left(\xi^{2}+\left(1+x^{2}\right)^{1 / 2} \mu_{j}\right)^{-s} d x d \xi
\end{aligned}
$$

The change of variable: $\xi \rightarrow \mu_{j}^{1 / 2} \xi$ leads to

$$
I(s)=\sum_{j=1}^{\infty} \mu_{j}^{-s+1 / 2}(2 \pi)^{-1} \iint\left(\xi^{2}+\left(1+x^{2}\right)^{1 / 2}\right)^{-s} d x d \xi .
$$


Moreover changing the variable $\xi \rightarrow\left(1+x^{2}\right)^{1 / 4} \xi$, we have

$$
I(s)=\sum_{j=1}^{\infty} \mu_{j}^{-s+1 / 2}(2 \pi)^{-1} \int_{-\infty}^{\infty}\left(1+x^{2}\right)^{-s / 2+1 / 4} d x \int_{-\infty}^{\infty}\left(1+\xi^{2}\right)^{-s} d \xi
$$

In combination with the well known equation

$$
\int_{0}^{\infty} \frac{x^{a}}{\left(1+x^{2}\right)^{1+b}} d x=\frac{\Gamma((a+1) / 2) \Gamma(b-(a-1) / 2)}{2 \Gamma(1+b)}
$$

when $\operatorname{Re} a, \operatorname{Re} b>-1$ and $\operatorname{Re} b>\operatorname{Re}(a-1) / 2$, we have

$$
I(s)=\frac{\Gamma(s / 2-3 / 4) \Gamma(s-1 / 2)}{2 \Gamma(s / 2-1 / 4) \Gamma(s)} \sum_{j=1}^{\infty} \mu_{j}^{-s+1 / 2}
$$

if $\operatorname{Re} s>3 / 2$.

Since $\Gamma(z)=1 / z-\gamma+O(z)$ as $z \rightarrow 0$ where $\gamma$ is the Euler number, we have

$$
\Gamma(s / 2-3 / 4)=\frac{1}{s / 2-3 / 4}-\gamma+O(s-3 / 2) \text { as } s \rightarrow 3 / 2 .
$$

Since

$$
G(s)=\frac{\Gamma(s-1 / 2)}{\Gamma(s / 2-1 / 4) \Gamma(s)}
$$

is holomorphic for $\operatorname{Re} s>1 / 2$ and $G(3 / 2)=2 \pi^{-1}$, we see that $G(s)=$ $2 \pi^{-1}+(s-3 / 2) G^{\prime}(3 / 2)+O\left((s-3 / 2)^{2}\right)$ as $s \rightarrow 3 / 2$. Therefore it follows that

$$
\frac{\Gamma(s / 2-3 / 4) \Gamma(s-1 / 2)}{\Gamma(s / 2-1 / 4) \Gamma(s)}=\frac{4 \pi^{-1}}{s-3 / 2}+\left\{2 G^{\prime}(3 / 2)-2 \gamma \pi^{-1}\right\}+O(s-3 / 2) .
$$

Using the fact which shall be proved in Appendix:

$$
\sum_{j=1}^{\infty} \mu_{j}^{-s+1 / 2}=\frac{1 / 2}{s-3 / 2}+C+O((s-3 / 2))
$$

where

$$
C=\frac{1}{2} \lim _{n \rightarrow \infty}\left[2 \sum_{k=1}^{n}(2 k-1)^{-1}-\log (2 n-1)\right]=(\gamma+\log 2) / 2,
$$

we have

$$
I(s)=\frac{\pi^{-1}}{(s-3 / 2)^{2}}+\frac{\left(G^{\prime}(3 / 2)-\gamma \pi^{-1}\right) / 2+2 C \pi^{-1}}{s-3 / 2}+R_{0}(s)
$$


where $R_{0}(s)$ is holomorphic for $\operatorname{Re} s>1 / 2$. In combination with well known equations

$$
\begin{aligned}
& \Gamma^{\prime}(1)=-\gamma, \quad \Gamma^{\prime}(3 / 2)=\pi^{1 / 2}+\Gamma^{\prime}(1 / 2) / 2 \text { and } \\
& \Gamma^{\prime}(1 / 2)=-\pi^{1 / 2}(\gamma+2 \log 2),
\end{aligned}
$$

it follows that we have

$$
\begin{aligned}
G^{\prime}(3 / 2) & =2 \Gamma^{\prime}(1) \pi^{-1}-\Gamma^{\prime}(1 / 2) \pi^{-3 / 2}-4 \Gamma^{\prime}(3 / 2) \pi^{-3 / 2} \\
& =(\gamma+6 \log 2-4) \pi^{-1} .
\end{aligned}
$$

Hence it turns out that

$$
I(s)=\frac{\pi^{-1}}{(s-3 / 2)^{2}}+\frac{(4 \log 2-2+\gamma) \pi^{-1}}{s-3 / 2}+R_{0}(s) .
$$

Thus by our Theorem, we have

$$
\begin{array}{r}
N_{A}(\lambda)=\frac{2}{3 \pi} \lambda^{3 / 2} \log \lambda+\frac{24 \log 2-16+6 \gamma}{9 \pi} \lambda^{3 / 2}+O\left(\lambda^{3 / 2-\delta}\right) \\
\text { as } \lambda \rightarrow+\infty .
\end{array}
$$

Appendix. In this appendix we shall consider the analytic continuation of

$$
Z(s)=\sum_{k=1}^{\infty}(2 k-1)^{-s}, \quad s=\sigma+i t
$$

where $\sigma$ and $t$ are real numbers. It is well known that $Z(s)$ is absolutely convergent for $\sigma>1$ and uniformly convergent for $\sigma \geq 1+\varepsilon$ for any $\varepsilon>0$. Then we shall give a proposition whose proof is essentially due to Siegel [12]

Proposition. $Z(s)$ can be continued analytically into the half-plane $\sigma>0$ and the continuation is holomorphic for $\sigma>0$, except for a simple pole $s=1$ with residue $1 / 2$. Further, $Z(s)$ has the expansion at $s=1$ :

$$
Z(s)-\frac{1 / 2}{s-1}=C+a_{1}(s-1)+a_{2}(s-1)^{2}+\cdots
$$

where

$$
C=\frac{1}{2} \lim _{n \rightarrow \infty}\left[2 \sum_{k=1}^{n}(2 k-1)^{-1}-\log (2 n-1)\right]=(\gamma+\log 2) / 2 .
$$


Before the proof of this proposition, we give

LEMMA. Let $f$ be a complex valued function belonging to $C^{1}[1,2 n-1]$. Then we have

$$
\begin{gathered}
\int_{0}^{2} \sum_{k=1}^{n-1} f^{\prime}(x+2 k-1)(x-1) d x+f(1)+f(2 n-1) \\
=2 \sum_{k=1}^{n} f(2 k-1)-\int_{1}^{2 n-1} f(x) d x
\end{gathered}
$$

Proof. Let $g$ be a complex valued function belonging to $C^{1}[0,2]$. Then, the integration by parts leads to

$$
\int_{0}^{2} g^{\prime}(x)(x-1) d x=g(0)+g(2)-\int_{0}^{2} g(x) d x .
$$

Letting $g(x)=f(x+2 k-1), k=1,2, \ldots, n-1$, it easily follows that $\int_{0}^{2} f^{\prime}(x+2 k-1)(x-1) d x=f(2 k-1)+f(2 k+1)-\int_{2 k-1}^{2 k+1} f(x) d x$. This completes the proof of Lemma.

Proof of Proposition. Let $f(x)=x^{-s}=e^{-s \log x}$ where $\log x$ takes the principal value. Then it follows from the above lemma that

$$
\begin{gathered}
-s \sum_{k=1}^{n-1} \int_{0}^{2}(x+2 k-1)^{-1-s}(x-1) d x+1+(2 n-1)^{-s} \\
=2 \sum_{k=1}^{n}(2 k-1)^{-s}-\int_{1}^{2 n-1} x^{-s} d x=F_{n}(s) .
\end{gathered}
$$

Here we easily see that

$$
F_{n}(s)=2 \sum_{k=1}^{n}(2 k-1)^{-s}-\frac{1-(2 n-1)^{1-s}}{s-1} \text { if } s \neq 1
$$

and

$$
F_{n}(s)=2 \sum_{k=1}^{n}(2 k-1)^{-1}-\log (2 n-1) \quad \text { if } s=1
$$

and therefore, it follows that $F_{n}(s)$ is an entire function of $s$. If $\sigma>1$, it follows that

$$
\int_{1}^{2 n-1} x^{-s} d x \rightarrow \frac{1}{s-1} \text { and } \sum_{k=1}^{n}(2 k-1)^{-s} \rightarrow Z(s) \text { as } n \rightarrow \infty
$$


Thus we see that $F_{n}(s)$ converges to $2 Z(s)-1 /(s-1)$. On the other hand, if $\sigma \geq \varepsilon>0$, it follows that the left-hand side in the above equality (A) converges to a holomorphic function for $\sigma>0$. Thus we see that $2 Z(s)-1 /(s-1)$ has the analytic continuation for $\sigma>0$. Let

$$
2 Z(s)-\frac{1}{s-1}=a_{0}+a_{1}(s-1)+a_{2}(s-1)^{2}+\cdots .
$$

Then it is easily seen that

$$
a_{0}=\lim _{n \rightarrow \infty}\left[2 \sum_{k=1}^{n}(2 k-1)^{-1}-\log (2 n-1)\right] .
$$

Finally a simple computation leads to

$$
\begin{aligned}
\sum_{k=1}^{2 n} \frac{1}{k}-\log (2 n)= & \frac{1}{2}\left\{\sum_{k=1}^{n} \frac{1}{k}-\log n\right\} \\
& +\left\{\sum_{k=1}^{n} \frac{1}{2 k-1}-\frac{1}{2} \log (2 n-1)\right\} \\
& +\frac{1}{2} \log \frac{n(2 n-1)}{4 n^{2}} .
\end{aligned}
$$

Noting that

$$
\lim _{n \rightarrow \infty}\left\{\sum_{k=1}^{n} \frac{1}{k}-\log n\right\}=\gamma(\text { the Euler constant }),
$$

we see that

$$
\lim _{n \rightarrow \infty}\left\{\sum_{k=1}^{n} \frac{1}{2 k-1}-\frac{1}{2} \log (2 n-1)\right\}=(\gamma+\log 2) / 2 .
$$

This completes the proof.

Acknowledgments. The author is indebted to the referee for a valuable suggestion, which led to a simpler reformulation of the main theorem.

\section{REFERENCES}

[1] J. Aramaki, Complex powers of a class of pseudodifferential operators and their applications, Hokkaido Math. J., XII No. 2 (1983), 199-225. asymptotic behavior of eigenvalues, Hokkaido Math. J., XVI No. 1 (1987), 1-28. On the asymptotic behaviors of spectrum of quasi-elliptic pseudodifferential operators on $\mathbf{R}^{n}$, to appear. 
[4] Complex powers of vector valued operators and its application to asymptotic behavior of eigenvalues, to appear.

[5] W. Donoghue, Distributions and Fourier Transforms, Academic Press, New York (1969).

[6] J. J. Duistermaat and V. W. Guillemin, The spectrum of positive elliptic operators and periodic bicharacteristics, Invention. Math., 29 (1975), 39-79.

[7] B. Helffer, Théorie spectrale pour des opérateurs globalement elliptiques, Société Math. de France, Astérique 1984.

[8] F. Menikoff and J. Sjöstrand, On the eigenvalues of a class of hypoelliptic operators, Math. Ann., 235 (1978), 55-85.

[9] A. Mohamed, Etude spectrale d'opérateurs hypoelliptiques à caractéristiques multiple, J. "Equation aux dérivées partielles" Saint Jean de Monts, juin 1981.

[10] D. Robert, Comportement asymptotique des valeurs propres d'opérateurs du type Schrödinger à potentiel dégénéré, J. Math. Pure et Appl., 61 (1982), 275-300.

[11] R. T. Seeley, Complex powers of an elliptic operator, Amer. Math. Soc. Symp. Pure Math., 10 (1967), 288-307.

[12] C. L. Siegel, Advanced analytic number theory, Tata Institute, 1980.

[13] N. Wiener, Tauberian theorems, Ann. of Math., 33 (1932), 1-100.

Received May 27, 1987 and in revised form August 17, 1987.

TOKYO DENKI UNIVERSITY

Hatoyama-Machi, Hiki-Gun, Saitama, 350-03, Japan 


\section{PACIFIC JOURNAL OF MATHEMATICS}

\section{EDITORS}

V. S. VARADARAJAN

(Managing Editor)

University of California

Los Angeles, CA 90024

Herbert Clemens

University of Utah

Salt Lake City, UT 84112

R. FINN

Stanford University

Stanford, CA 94305
HERMANN FLASCHKA

University of Arizona

Tucson, AZ 85721

RAMESh A. GANGOLLI

University of Washington Seattle, WA 98195

VAUGHAN F. R. JONES

University of California

Berkeley, CA 94720
ROBION KIRBY

University of California

Berkeley, CA 94720

C. C. MOORE

University of California

Berkeley, CA 94720

HAROLD STARK

University of California, San Diego

La Jolla, CA 92093

\section{ASSOCIATE EDITORS}
R. AREnS
E. F. BECKENBACH
B. H. NEUMANN
F. WOLF
K. YOSHIDA (1906-1982)

\section{SUPPORTING INSTITUTIONS}

UNIVERSITY OF ARIZONA

UNIVERSITY OF OREGON

UNIVERSITY OF BRITISH COLUMBIA UNIVERSITY OF SOUTHERN CALIFORNIA

CALIFORNIA INSTITUTE OF TECHNOLOGY

UNIVERSITY OF CALIFORNIA

STANFORD UNIVERSITY

MONTANA STATE UNIVERSITY

UNIVERSITY OF HAWAII

UNIVERSITY OF NEVADA, RENO

UNIVERSITY OF TOKYO

NEW MEXICO STATE UNIVERSITY

UNIVERSITY OF UTAH

OREGON STATE UNIVERSITY

WASHINGTON STATE UNIVERSITY

UNIVERSITY OF WASHINGTON 


\section{Pacific Journal of Mathematics}

\section{Vol. 133, No. $1 \quad$ March, 1988}

John Anderson, Finitely generated algebras and algebras of solutions to partial differential equations $\ldots \ldots \ldots \ldots \ldots \ldots \ldots \ldots \ldots \ldots \ldots \ldots \ldots$

Junichi Aramaki, On an extension of the Ikehara Tauberian theorem . . . . . 13

Giacomo Monti Bragadin, Abstract Riemannian stratifications .......... 31

Lawrence James Brenton and Richard Hill, On the Diophantine equation

$1=\sum 1 / n_{i}+1 / \prod n_{i}$ and a class of homologically trivial complex

surface singularities .................................41

C. Bruce Hughes, Controlled homotopy topological structures $\ldots . \ldots \ldots \ldots 69$

Peter Wilcox Jones and Takafumi Murai, Positive analytic capacity but zero Buffon needle probability . ...........................999

Gary M. Lieberman, Hölder continuity of the gradient at a corner for the

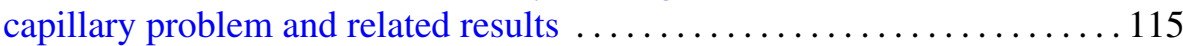

Feng Luo, Representing homology classes of $C \mathbf{P}^{2} \overline{C \mathbf{P}^{2}}$

Claudio Nebbia, Groups of isometries of a tree and the Kunze-Stein

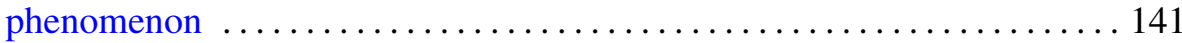

Stefan Richter, Unitary equivalence of invariant subspaces of Bergman and

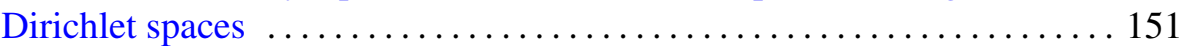

Paul Frederick Ringseth, The Selberg trace formula for groups without Eisenstein series ..................................... 157

Abderrazzak Sersouri, The Mazur property for compact sets $\ldots \ldots \ldots \ldots 185$ Alladi Sitaram, On an analogue of the Wiener Tauberian theorem for symmetric spaces of the noncompact type 